Protocol

\title{
Development of a Methodology for Estimating the Availability of ADAS-Dependent Road Infrastructure
}

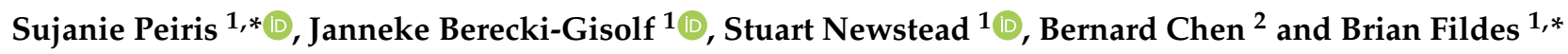 \\ 1 Accident Research Centre, Monash University, 21 Alliance Ln, Clayton, VIC 3800, Australia; \\ janneke.berecki-gisolf@monash.edu (J.B.-G.); stuart.newstead@monash.edu (S.N.) \\ 2 Department of Mechanical and Aerospace Engineering, Monash University, 17 College Walk, \\ Clayton, VIC 3800, Australia; Bernard.Chen@monash.edu \\ * Correspondence: Sujanie.Peiris@monash.edu (S.P.); Brian.Fildes@monash.edu (B.F.); Tel.: +61-3-9905-9979
}

Citation: Peiris, S.; Berecki-Gisolf, J.; Newstead, S.; Chen, B.; Fildes, B. Development of a Methodology for Estimating the Availability of ADAS-Dependent Road Infrastructure. Sustainability 2021, 13, 9512. https://doi.org/10.3390/ su13179512

Academic Editor: Anna Granà

Received: 24 June 2021

Accepted: 18 August 2021

Published: 24 August 2021

Publisher's Note: MDPI stays neutral with regard to jurisdictional claims in published maps and institutional affiliations.

Copyright: (c) 2021 by the authors. Licensee MDPI, Basel, Switzerland. This article is an open access article distributed under the terms and conditions of the Creative Commons Attribution (CC BY) license (https:// creativecommons.org/licenses/by/ $4.0 /)$.

\begin{abstract}
Advanced driver assist systems are being promoted with the expectation that enhanced driver support will mitigate road trauma. While these technologies are optimised for certain road and traffic conditions, not all roads across Australasia are equipped with ADAS-supportive infrastructure. This study developed a desk-top methodology for using road classes (disaggregated by remoteness levels) to estimate the presence of quality roads, road delineation and speed signage in Victoria, Australia. Aerial imagery and mapping data were used to assess a number of random locations based on a developed protocol. The methodology demonstrated that in Victoria, major and arterial roads across all remoteness levels had high-quality sealed surfaces but $42 \%$ of all remote roads were unsealed. Delineation (crucial for lane support systems) were absent across $73 \%$ of sub-arterial roads independent of remoteness, and absent across $96 \%$ of sub-arterial roads in regional and remote areas. Speed sign availability across remote and regional areas was sparse, with only $65 \%$ of all roads assessed having signage. Results are reflective of Victoria's road funding model and consistent with on-road audits conducted by other researchers. This methodology enables the proportion ADAS-ready roads to be estimated so the benefits of ADAS technologies can be quantified and investments into ADAS-supportive infrastructure be readily allocated.
\end{abstract}

Keywords: ADAS; safety; infrastructure; delineation; speed signage

\section{Introduction \\ 1.1. Importance of ADAS Technologies}

Given that over $90 \%$ of road crashes are considered to be a result of human error [1-4], vehicle-related interventions are expected to provide significant road safety gains [5-9]. Advanced driver assist systems (ADAS) help drivers manage their speed and remain in control of vehicle direction, eliminating or mitigating the risk of death or serious injury by reducing crash likelihood. ADAS technologies which address driver inattention, fatigue and error include but are not limited to lane keep assist (LKA) and its less sophisticated variant, lane departure warning (LDW), intelligent speed adaptation (ISA) and autonomous emergency braking (AEB) [10]. It is implied that given the increasing fitment rates of these technologies [11,12], they will be relied upon as crash-mitigation tools across road networks.

The functionality or accuracy of ADAS features is heavily reliant on the presence of adequate road infrastructure which is not always available. A combination of sensors (Table 1) built into the vehicle, continuously scan the environment for available infrastructure to ensure correct ADAS function. In regions like Australasia, new vehicle ownership in regional and remote areas are comparable to that of major cities [13], yet infrastructure availability is not consistent across remoteness levels. So while the relationship between ADAS technologies and existing road infrastructure appears highly compatible in some parts of the world [14], these findings are not resonated in Australasia [15]. 
Table 1. Overview of sensors fitted into new vehicles and their limitations, obtained from Austroads (2020) by Somers (2019), p. 8.

\begin{tabular}{|c|c|c|}
\hline Sensor & Infrastructure Sensed & Limitations \\
\hline Camera-based machine vision & $\begin{array}{c}\text { Scene semantics: } \\
\text { Signs/lines/traffic signals/stop lines }\end{array}$ & $\begin{array}{l}\text { Line of sight } \\
\text { Sun glare } \\
\text { Poor weather }\end{array}$ \\
\hline LiDAR & $\begin{array}{c}\text { Fixed objects such as buildings, signs, concrete } \\
\text { barriers. Often used with mapping to } \\
\text { localise vehicles. } \\
\text { Can detect line markings through reflectivity }\end{array}$ & $\begin{array}{c}\text { Line of sight } \\
\text { Poor weather } \\
\text { Poor colour detection } \\
\text { Cost of sensors/power required to process LiDAR }\end{array}$ \\
\hline Radar & $\begin{array}{l}\text { Fixed infrastructure such as concrete barriers } \\
\text { and buildings }\end{array}$ & Radio frequency interference \\
\hline ADAS/HD Maps & $\begin{array}{l}\text { Used with Global Navigation Satellite System } \\
\text { (GNSS), LiDAR or radar/machine vision to } \\
\text { localise vehicles, provide path } \\
\text { awareness/planning and provide a detailed } \\
\text { map of attributes the vehicle 'expects' to see }\end{array}$ & $\begin{array}{l}\text { Construction on roadways effectively changes } \\
\text { features on the maps } \\
\text { Significant cost to map areas and keep maps } \\
\text { updated with changes }\end{array}$ \\
\hline Connectivity & $\begin{array}{c}\text { Primarily cellular for network } \\
\text { coverage/availability, and required for beyond } \\
\text { line of sight sensing on the vehicle. } \\
\text { Changed conditions e.g., roadworks, changes } \\
\text { to speed zones, road hazards, } \\
\text { GNSS augmentation }\end{array}$ & $\begin{array}{l}\text { Network coverage } \\
\text { Latency/packet loss } \\
\text { Security }\end{array}$ \\
\hline Ultrasound & $\begin{array}{l}\text { Nil } \\
\text { Used for immediate local detection in } \\
\text { immediate vehicle range }\end{array}$ & Short range \\
\hline GNSS (Augmented/not augmented) & $\begin{array}{c}\mathrm{Nil} \\
\text { Used for positioning and localisation }\end{array}$ & $\begin{array}{c}\text { Tunnels, urban canyons, spoofing, overall } \\
\text { low accuracy. } \\
\text { For ADAS and automated driving, highly accurate } \\
\text { GNSS is desirable }(5-20 \mathrm{~cm}) \text {. To achieve this } \\
\text { cellular coverage, ground station corrections are } \\
\text { used to augment the signal }\end{array}$ \\
\hline
\end{tabular}

\subsection{Deficiencies in Current Road Infrastructure}

Despite the reliance which new vehicle technologies have on road infrastructure, there is no single agency in Australasia who is responsible for maintaining an inventory of road infrastructure data. This makes it near impossible to quantify the likely benefits of the technologies, estimate forgone benefits or target investments which will see the technologies be better supported in future. Road infrastructure such as quality sealed surfaces, contrasting and wide delineation and machine-detectable speed signs, for example, are some prerequisites for AEB, LDW or LKA and ISA function respectively. For vehicle mounted cameras (which assist with LDW or LKA), the presence of high contrasting delineation (i.e., edgeline and centreline) is essential, noting sensitivities of the cameras to adverse weather, light and certain road geometries [16-19]. More advanced lane-support systems are predicted to be less sensitive to the presence of edgelines, however these systems are rare. Speed sign-recognition cameras, which are paramount for ISA function are also at the mercy of infrastructure. These cameras are sensitive to the position, placement, size, angle and design of speed signs [20]. Since Australia does not as yet have digital HD speed data maps or GPS-linked speed limit databases, and given the inconsistent cellular coverage across the nation [15], the functionality and reliability of ISA is largely dependent on the presence of physical road signs.

\subsection{On-Road Trials}

In recognition of the need to assess the availability and quality of existing road infrastructure, on-road assessments of connected and autonomous vehicles (CAVs, i.e., SAE Level 4 automation and above) and ADAS technologies are being undertaken globally, and specifically in every Australian State, Territory and in New Zealand [21,22]. The majority of these trials involve the use of automated shuttlebuses operating in pre-defined loops [23-26], mobility service trials in designated areas [27], CAV studies that look at 
optimising vehicle-to-vehicle and vehicle-to-infrastructure interaction $[28,29]$ and a number of on-road audits and trials.

The most notable of these audits, conducted by Austroads was to gauge the availability and machine legibility of road infrastructure on key high traffic urban freeways, rural freeways and key rural highways across Australasia $[15,30]$. The quality, width, contrast and retro-reflectivity of line markings, speed limit and traffic restriction signage, temporary changes to road conditions and availability of digital map data were identified with respect to being CAV-ready and ADAS supportive. The research highlighted that while most freeways and highways in Australasia could support ADAS technologies with regards to lane positioning and cellular availability, the majority of local roads could not. Speed limit signs were reliably detected and read using automated technology provided they were in the correct positions. Given the high incidence of roadworks and temporary changes to roads, it was concluded that CAV operation on the network would be challenging even if high definition maps were available in Australasia. The audit, although thorough and used real-time machine vision systems to sample $25,000 \mathrm{~km}$ of roads, was based on best-case conditions (away from complicated intersections and developed areas) and sampled less than $2 \%$ of the total network $[15,30]$.

An on-road trial using ten vehicles (from nine different vehicle manufactures) conducted by Transurban [31] explored how ADAS fitted vehicles performed within Sydney's Orbital network $(7110 \mathrm{~km})$. Lane keep assist was found to be sensitive to long cracks along bitumen sealed surfaces, while road markings (other than delineation) and changes in the road surface were found to disengage lane keeping. Consistent with the findings in literature, lane detection cameras were found to be sensitive to changes in road surface and lighting, sharp road curvature-all affecting LKA performance and engagement. The functionality of traffic sign recognition was influenced by the type of sign available and the location/position of the signs. Furthermore, the trial found that ISA systems that relied on digital speed maps occasionally identified changes in speed when there were none. Almost identical findings were published by an equivalent Transurban trial [32] conducted in Victoria over $4900 \mathrm{~km}$ of high-quality freeways.

To date, no studies have published findings regarding the performance of ADAS or CAV in regional and remote locations specifically, although it should be acknowledged that these studies are currently in progress [25,33]. While Mackenzie et al. (2018) drove two vehicles equipped with LDW technology over $155 \mathrm{~km}$ of delineated rural Highway in Western Australia, the authors did not investigate delineation availability. The researchers found that of the 189 crossing events triggered, LDW accurately warned against $81 \%$ of these. Failures were attributed to the absence of marked lines, the vehicle travel speed being less than the manufacturer-recommended operation speed for the system to function, line markings being difficult to detect, poor retro-reflectivity and daylight brightness being too low for the system settings.

Literature and on-road trials provide extensive evidence that ADAS technologies are highly dependent on road infrastructure. Yet, despite the prevalence of these technologies in the current fleet and continued push for vehicle manufactures to provide the technologies as standard [34], few efforts have been made to quantify the availability of technologysupportive infrastructure. In Australasia, given the absence of consistent road asset data, assessing and quantifying the vastly diverse $870,000 \mathrm{~km}$ of roads for 'ADAS-readiness' are not trivial tasks. Alternative infrastructure quantification methods are needed.

The current study aimed to develop a framework by which road classes could be used to estimate the presence of sealed roads, adequate delineation and speed signs across Australia and New Zealand. No evidence was found in the literature of such an approach undertaken to date. By establishing a method which could quantify the presence of appropriate road infrastructure in Victoria, it is anticipated that Australasia's road-readiness for ADAS technologies can be established. 


\section{Materials and Methods}

The distribution of fatal and serious injury (FSI) crashes in Victoria (2013-2018) were mapped by road class and disaggregated by remoteness level. Based on this distribution, a number of random locations were identified using GPS coordinates through an in-built function in ArcGIS software (ArcGIS Software is a commercial product by ESRI for working with maps and geographic information, Section 2.3). These locations were visited virtually using Nearmap (Nearmap Ltd. is an Australian aerial imagery technology company with location data that provides frequently-updated, high-resolution aerial imagery) and the presence and quality of infrastructure identified. Protocol was developed to assess the quality of the existing road surface, and the quality/presence of delineation. A speed sign map was then overlaid onto the Victorian road network to determine the distance from each random point to available speed signs. Figure 1 outlines the methodology which was employed to assess road surface quality, delineation availability and speed sign availability in Victoria.

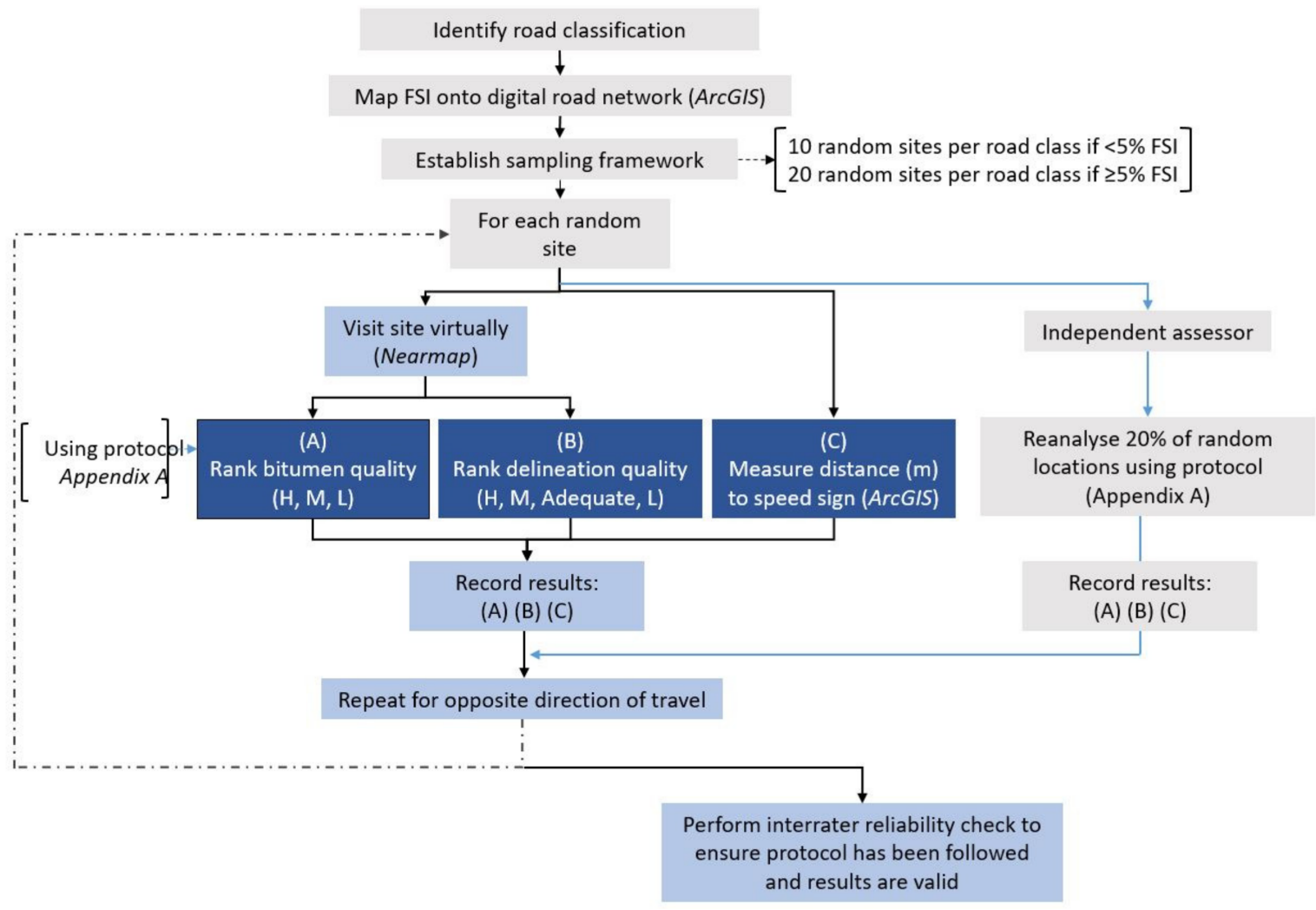

Figure 1. A schematic of the methodology which was employed to assess road attributes (surface quality, delineation availability and speed sign availability) across road classes, Victoria.

\subsection{Remoteness Structure}

Remoteness structure identified by the Australian Statistical Geography Standard (ASGS) was used to categorise roads as being in either major cities, regional (i.e., 'Inner regional' or 'Outer regional') or remote (i.e., 'Remote' or 'Very remote') areas [35]. The ASGS standard identifies the level of remoteness based on a measure of relative access to services.

\subsection{Road Network}

In Australia, the declared road network comprises approximately $15 \%$ of the entire road network and consists of Freeways, Arterial-Highways, Other-Arterials, Local roads, Non-Arterial State roads, Other Roads and Proposed Committed roads. However, since the undeclared network is not mapped on the map of declared roads, an alternative 
road classification for random sampling was sought. Ultimately, the 'Victorian Road Network-Vicmap Transport' was used for establishing a random sampling framework since this allowed local roads, collector roads and sub-arterials to be identified and included in the analysis. An extensive digital road network map containing line features and distinguishing freeways, highways, arterial roads, sub-arterials, connectors, local roads, bridges, footbridge, ferry routes, foot tracks, $4 \mathrm{wd}$ tracks, roundabouts and tunnels (last updated 21 February 2020), was used [36]. The GDA94 projection system was used to import the road network for Victoria.

\subsection{Identifying a Sampling Framework}

The GPS coordinates of fatality and serious injury (FSI) crashes in Victoria (2013-2018) were mapped onto freeways, highways, arterial roads, sub-arterial roads, collector roads and local roads and further disaggregated by remoteness, excluding FSI which involved heavy vehicles (GMV $\geq 4500 \mathrm{~kg}$ ). Clipping was performed in ArcGIS using an $\mathrm{x}-\mathrm{y}$ tolerance of $3 \mathrm{~m}$ to capture as many crashes as possible onto the road network. Based on the distribution of FSI crashes across the road classes, a number of random locations were selected using an in-built function in ArcGIS. Ten random locations were chosen for the assessment per road class if less than $<5 \%$ of FSI crashes occurred on a road class and remoteness level. Twenty random samples were chosen for the analysis if $>5 \%$ of FSI crashes occurred on a road class and remoteness level.

\subsection{Road Surface Quality and Delineation Quality Protocol}

The analysis for each site included looking at number of characteristics which allowed for the quantification of surface quality and appropriate machine detectable delineation. All data were acquired using Nearmap satellite imagery, using aerial (birdseye) view and then verified using street-view as the latter provides a higher resolution. The variables were collected for each random location identified by ArcGIS. Fields of interest and an explanation of factors considered for each field of interest is presented as Appendix A.

All data were collected by one individual to ensure consistency in judgement of road characteristics during the data collection process. To ensure consistency of the coding protocol, interrater reliability was assessed for $20 \%$ of locations through review by an independent assessor using the assessment protocol (Appendix A). The interrater reliability was calculated using the kappa statistic.

\subsubsection{Road Surface Quality}

Road surface quality was ranked as High, Medium or Low based on vehicles ability to come to a complete stop without loss of control in the event that a driver braked suddenly in a hypothetical emergency situation. Each section of road was assessed from a driver's perspective, looking ahead as far as the image allowed, again using aerial view and then verified using street-view in Nearmap. The quality of bitumen was assessed based on the presence of alligator and longitudinal cracks and general defects including longitudinal depressions in wheel path, pavement drop-offs, transverse undulations of pavement surface, visible friction variations and potholes.

The following grades were assigned for each road during the assessment:

High-Roads which had a fully sealed surface, with bitumen appearing smooth, fully intact and well maintained.

Medium-Roads which were sealed but the bitumen appeared to be highly worn, cracked or not well maintained with differences in friction along the length of the surface clearly visible. These roads were adequately sealed to accommodate sudden breaking.

Low-Roads which were unsealed roads or where road seal appeared to be of poor quality with severe defaults and unmaintained, where grip between a vehicle's tyres and the road would be compromised in adverse weather conditions if sudden braking was required. 
The roads were rated for one direction of travel, then moving onto the opposing lane, assessed for the second direction of travel.

\subsubsection{Delineation Quality}

Delineation quality was assessed across the road network, assuming a vehicle with LSS activate was attempting to maintain its path within a lane. On multilane roads (such as freeways, highways, arterial roads), delineation quality was assessed assuming a vehicle fitted with LSS was travelling straight through on the left-most lane. This provided the most conservative gauge of delineation presence since on multilane roads, vehicles travelling in the central lanes are likely to have adequate lane markings to keep within the lane while those travelling in the left-most ('straight-ahead') lane do not always preview lane markings depending on the standard of the road.

Given that manufacturer requirements stipulate that lane keep technologies are only designed to function at speeds of $60 \mathrm{~km}$ or above [37], local roads and collector roads were excluded from this analysis. Local roads in Victoria are signed at $50 \mathrm{~km}$ or below and collector roads are signed at $60 \mathrm{~km} / \mathrm{h}$ [38] where lane keep technologies are unlikely to be of benefit, independent of delineation presence or quality. Further, Victoria has no region defined as being 'Very Remote' and within Remote Victoria, there were no roads classified as Freeways and therefore, no such roads could be sampled.

Road delineation quality was broken down into four categories based on the presence, consistency, clarity and contrast of delineation against the underlying bitumen, the presence and contrast (including texture, colour and consistency) of shoulders and engineered gutters abutting the bitumen. Rankings of High, Medium, Adequate and Low were assigned as follows:

High-Roads which had high-quality sealed bitumen surfaces, high contrasting edgelines and centrelines present. These roads were likely to meet the conditions specified by the most basic LKA protocol tests conducted by EuroNCAP and ANCAP (i.e., solid-paved surface with a maximum slope of $1 \%$ in the longitudinal direction, $<2 \%$ for half a lane width either side of the centreline and $<3 \%$ for the outer half of the test lane in lateral direction.... dashed line with a width between 0.10 and $0.25 \mathrm{~m}$ or 0.10 and $0.15 \mathrm{~m}$ for centrelines, solid line with a width between 0.10 and $0.25 \mathrm{~m}$, road edge consisting of grass and/or gravel or approved surrogate) [39])

Medium-Roads which had a bitumen surface that could be considered high or medium quality and had delineation that was consistently present and clearly visible, and likely to be detected by machine seeing systems. These roads were not necessarily of the highest class and could potentially lead to the detection of false edges if there were longitudinal cracks in the bitumen, but assuming fitment of advanced LSS technologies, would likely be supportive of lane keep functions. In such locations, either both edgelines and centrelines were present, or had a centreline and high contrasting guttering which would likely be detected by sensors in lieu of an edgeline.

Adequate-Roads where at present, the majority of LSS are unlikely to perform due to there being an absence of an edgeline and/or centreline, however, have a gutter or visually contrasting shoulder present (of high or medium contrast) on either side of the travel lane, which could potentially be detected by machine seeing systems as lane markings. Evidence from testing organisations suggest that lane support systems can, depending on the precision of the technology, keep within a lane in the absence of edgeline, provided adequately contrasting material is present abutting the bitumen [40].

Low-Roads, which were independent of how advanced lane keep technologies became would not be supportive of LSS technologies given they are either entirely unsealed roads or roads which had bitumen surfaces with no delineation, no highly contrasting shoulders and had either no roadside gutters available or had roadside gutters but no centreline which would allow for the vehicle to remain within a lane. 


\subsection{Speed Sign Availability}

In the absence of digital speed maps, vehicles will rely largely on speed sign detection technologies to advise drivers of the enforced speed limits. Therefore, the distance to speed signs was measured from each random location, assuming one direction of travel in the first instance, and then the opposite direction of travel. Speed sign data generated and made available by Data VIC [41] were overlaid on the Victorian road network map. Measurements were made in ArcGIS and verified using Google Maps. The distances were then averaged for each road type and remoteness level to identify the frequency of speed sign locations based on road class and remoteness. In the absence of speed maps, drivers would have to travel these distances on average before the cameras could identify a speed sign.

\section{Results}

\subsection{Sampling Framework}

The FSI crashes in Victoria (2013-2018) were mapped onto the road network (Table 2) to determine the number of random locations to assess (Table 3).

Table 2. Percentage distribution of fatal and serious injury crashes in Victoria by the six main road classifications and remoteness levels.

\begin{tabular}{cccc}
\hline & Major Cities & Regional & Remote \\
\hline Freeways & 4.96 & 1.06 & Not available \\
Highway & 7.92 & 4.95 & 0.08 \\
Arterial & 19.83 & 5.89 & 0.00 \\
Sub-arterial & 13.87 & 4.22 & 0.00 \\
Collector & 8.78 & 1.30 & 0.00 \\
Local & 20.42 & 6.69 & 0.03 \\
\hline
\end{tabular}

Table 3. Number of locations sampled across the road network, based on the distribution of FSI crashes, VIC.

\begin{tabular}{cccc}
\hline & Major Cities & Regional & Remote \\
\hline Freeways & 10 & 10 & Not available \\
Highway & 20 & 10 & 10 \\
Arterial & 20 & 20 & 10 \\
Sub-arterial & 20 & 10 & 10 \\
Collector & 20 & 10 & 10 \\
Local & 20 & 20 & 10 \\
\hline
\end{tabular}

\subsection{Road Quality}

The following cross tabulation (Table 4) shows the distribution of high, medium and low quality roads across Victoria by road class and remoteness, and independent of remoteness level (Table 5). 
Table 4. Percentage of roads with high, medium and low surface quality roads by road class and remoteness, VIC (percentage calculations are with respect to each remoteness level).

\begin{tabular}{ccccc}
\hline & & High & Medium & Low \\
\hline & Freeways & 7.8 & 0.9 & 0.0 \\
& Highway & 16.0 & 2.3 & 0.0 \\
Major City & Arterial & 12.3 & 5.9 & 0.0 \\
$(100 \%)$ & Sub-arterial & 13.2 & 4.6 & 0.5 \\
& Collector & 14.6 & 2.7 & 0.9 \\
& Local & 15.5 & 1.4 & 1.4 \\
\hline & Freeways & 12.5 & 0.0 & 0.0 \\
Regional & Highway & 10.6 & 1.9 & 0.0 \\
$(100 \%)$ & Arterial & 16.9 & 7.5 & 0.6 \\
& Sub-arterial & 1.3 & 2.5 & 8.8 \\
& Collector & 1.3 & 2.5 & 17.5 \\
\hline & Local & 2.5 & 5.0 & NA \\
& Freeways & NA & NA & 0.0 \\
Remote & Highway & 19.0 & 1.0 & 2.0 \\
$(100 \%)$ & Arterial & 10.0 & 8.0 & 4.0 \\
& Sub-arterial & 10.0 & 6.0 & 20.0 \\
& Collector & 0.0 & 0.0 & 16.0 \\
\hline
\end{tabular}

Table 5. Road surface quality in Victoria by road network independent of remoteness level, VIC.

\begin{tabular}{ccccc}
\hline & & High & Medium & Low \\
\hline \multirow{6}{*}{ VIC (overall) } & Freeways & 7.7 & 0.4 & 0.0 \\
& Highway & 14.8 & 1.9 & 0.0 \\
& Arterial & 13.4 & 6.9 & 0.6 \\
& Sub-arterial & 8.6 & 4.2 & 4.0 \\
& Collector & 7.1 & 2.1 & 7.5 \\
& Local & 7.9 & 3.1 & 9.8 \\
\hline
\end{tabular}

\subsection{Road Delineation Quality}

The following tabulation (Table 6) shows the availability of roads with high, medium, adequate and low quality delineation within Victoria, broken down by road class and remoteness. Delineation quality across road classes, independent of remoteness levels, is presented in Table 7.

Table 6. Percentage of roads with high, medium, adequate and low delineation by road class and remoteness, VIC.

\begin{tabular}{cccccc}
\hline & & High & Medium & Adequate & Low \\
\hline \multirow{4}{*}{ Major Cities } & Freeways & 11.4 & 1.4 & 1.4 & 0.0 \\
& Highway & 11.4 & 7.9 & 7.1 & 2.1 \\
& Arterial & 6.4 & 11.4 & 4.3 & 6.4 \\
& Sub-arterial & 13.6 & 5.0 & 1.4 & 8.6 \\
\hline \multirow{5}{*}{ Regional Roads } & Freeways & 19.0 & 1.0 & 0.0 & 0.0 \\
& Highway & 3.0 & 16.0 & 1.0 & 0.0 \\
& Arterial & 21.0 & 4.0 & 3.0 & 12.0 \\
& Sub-arterial & 2.0 & 0.0 & 0.0 & 18.0 \\
\hline \multirow{5}{*}{ Remote Roads } & Freeways & NA & NA & NA & NA \\
& Highway & 18.3 & 11.7 & 0.0 & 3.3 \\
& Arterial & 3.3 & 6.7 & 13.3 & 10.0 \\
\cline { 2 - 5 } & Sub-arterial & 0.0 & 0.0 & 0.0 & 33.3 \\
\hline
\end{tabular}


Table 7. Delineation quality in Victoria by road class independent of remoteness, VIC.

\begin{tabular}{ccccc}
\hline VIC (Overall) & High & Medium & Adequate & Low \\
\hline Freeway & 11.7 & 1.0 & 0.7 & 0.0 \\
Highway & 10.0 & 11.3 & 3.7 & 1.7 \\
Arterial & 10.7 & 8.0 & 5.7 & 9.0 \\
Sub Arterial & 7.0 & 2.3 & 0.7 & 16.7 \\
\hline
\end{tabular}

\subsection{Speed Sign Availability}

The following table (Table 8) provide the average distance to speed signs from random locations within each road class and remoteness level. If speed signs were unavailable on a length of road, these road sections were measured. Local roads and some collector roads were not measured since these roads were often unsigned.

Table 8. Speed sign availability, Victoria.

\begin{tabular}{|c|c|c|c|c|c|c|}
\hline & & Sites Examined & $\begin{array}{l}\text { No. of Signs } \\
\text { Available }\end{array}$ & $\begin{array}{l}\text { Av. Distance from } \\
\text { Random Location to } \\
\text { Sign (m) }\end{array}$ & $\begin{array}{l}\text { No. of Roads } \\
\text { with No Signs }\end{array}$ & $\begin{array}{c}\text { Av. Length of } \\
\text { Road with No } \\
\text { Sign (m) }\end{array}$ \\
\hline \multirow{6}{*}{ Major City (100\%) } & Freeways & 20 & 20 & 1280 & 0 & - \\
\hline & Highway & 40 & 40 & 230 & 0 & - \\
\hline & Arterial & 40 & 40 & 185 & 0 & - \\
\hline & Sub-arterial & 40 & 38 & 305 & 2 & 9800 \\
\hline & Collector & 40 & 31 & 310 & 9 & 1090 \\
\hline & Local & 20 & 4 & 980 & 16 & NM \\
\hline \multirow{6}{*}{$\begin{array}{l}\text { Regional } \\
(100 \%)\end{array}$} & Freeway & 20 & 20 & 1860 & 0 & - \\
\hline & Highway & 20 & 18 & 13,660 & 2 & 25,000 \\
\hline & Arterial & 40 & 37 & 9285 & 3 & 86,350 \\
\hline & Sub Arterial & 20 & 6 & 24,421 & 14 & 9065 \\
\hline & Collector & 20 & 12 & 1500 & 8 & 2255 \\
\hline & Local & 20 & 0 & NA & 20 & NM \\
\hline \multirow{6}{*}{$\begin{array}{c}\text { Remote } \\
(100 \%)\end{array}$} & Freeway & NA & NA & NA & & \\
\hline & Highway & 20 & 20 & 7385 & - & \\
\hline & Arterial & 20 & 14 & 14,970 & 6 & 7170 \\
\hline & Sub Arterial & 20 & 2 & 17,535 & 18 & 10,845 \\
\hline & Collector & 20 & 2 & 5100 & 18 & NM \\
\hline & Local & 20 & 0 & NA & 20 & NM \\
\hline
\end{tabular}

$\mathrm{NM}=$ not measured, NA = not available.

\subsection{Interrater Reliability}

Interrater reliability or measure of agreement for road surface quality was measured using the Kappa statistic $(\mathrm{k}=0.786, \mathrm{SE}=0.062, p<0.000)$ and indicated substantial level of agreement between raters. The Kappa statistic for road delineation quality $(k=0.812$, $\mathrm{SE}=0.049, p<0.000)$ indicated almost perfect agreement between raters [42].

\section{Discussion}

This study developed a desktop analysis method for estimating the proportion of roads which are fitted with AEB, LSS and ISA-necessary infrastructure based on road class and remoteness level. By using Victoria as a case study, road classes (further disaggregated by remoteness level) were randomly sampled using aerial imagery to establish road quality. Road classes in Victoria were successfully used to identify the proportion of roads which have high-quality sealed surfaces, adequate delineation and identify the proportion of roads with an adequate density of speed signs (supportive of ISA function). The findings are consistent with and validated by the extensive on-road field trials and audits conducted in Australasia [15,31,32,43-45]. This methodology enables practitioners and researchers to use road class systems and remoteness levels to cost-effectively determine their level of preparedness to support highly prevalent ADAS technologies.

\subsection{Road Surface Quality}

Road surface type and condition effects road safety, vehicle operating costs and noise generation with road texture specifically influence skid and rolling resistance [46]. Here, 
road surface quality was assessed at a macro-level. Roads with high surface quality were most prevalent on freeways, highways and arterial roads, consistent with operational responsibility. Local roads in regional and remote areas appeared to have the worst surface quality (reflective of approximately $22 \%$ or $33,000 \mathrm{~km}$ of the Victorian road network). These roads had questionable road friction and were unlikely to be supportive in the event that sudden braking (or AEB) was needed (predominantly sub-arterial roads, collector roads and local roads in regional and remote Victoria). The results are consistent with those reported in the 2018 ALGA report [47] and by BITRE [48]. BITRE notes that of the $874,000 \mathrm{~km}$ of road length across Australia, only 381,000 km (less than $44 \%$ ) are fully paved roads, with the remaining $56 \%$ being unsealed. Of the total length of unsealed roads, the State of the Assets Report [47] by the Australian Local Governments Association found that $53 \%$ are rated poor to fair quality (as per International Infrastructure Management Manual rating scale [49]), suggestive that $30 \%$ of the road network was unlikely to be supportive of current traffic volumes and future vehicle technologies. Comparable to the $22 \%$ identified using this methodology, the results suggest that at least one-fifth of the network is unlikely to have any basic road infrastructure, let alone being technology-supportive.

At present surface inspection ratings (SIR) have become a standardised system for assessing pavement surface condition for sprayed seal and asphalt roads across Australasia [50]. The financial and time intensive technique systematically prioritises roads for resurfacing based on available funding. With the demarcation of operational responsibility being identified by the Road Management Act in 2004, it is ensured that freeways and arterial roads in urban and non-urban areas are typically monitored and maintained. Not surprisingly, this methodology identified that in Victoria, 98.7\% of freeways, highways and arterial roads had high-medium quality surfaces, an almost-identical finding to the on-road audit conducted by Austroads [15,44]. Likely reflective of the road management model, only $60 \%$ of sub-arterial roads, collector roads and local roads were sealed or had high-medium quality surfaces.

\subsection{Delineation Quality}

Enhancing visibility of lane delineation is typically considered to have positive effects on road safety and consequently incorporated into best practices globally (the U.S. Manual on Uniform Traffic Control Devices, [51]). Here, roads with the highest delineation quality were considered to be those with high contrasting delineation and non-homogenous edges abutting bitumen. Other than improving general safety, these features are specifically recommended for optimal performance of current generation LSS systems [52]. Of the four Victorian road classes examined, delineation quality was found to be greatest on freeways and highways independent of remoteness level while almost half of arterial roads and sub-arterial roads lacked high-quality road markings. Translating this to road lengths using mapping data suggests that of the total Victorian road network (almost $151,000 \mathrm{~km}$ in length), 41,350 km should have high- to medium-quality delineation given these roads are signed at $70 \mathrm{~km} / \mathrm{h}$ or faster. Freeways and highways constitute almost $4600 \mathrm{~km}$ of this network, with $3900 \mathrm{~km}(85 \%)$ having high- or medium-quality markings. Sub-arterial roads and arterial roads constitute almost $36,800 \mathrm{~km}$ of the Victorian road network however, less than half $(17,150 \mathrm{~km}, 46.6 \%)$ had the same delineation contrast. The results presented here resonate with the findings of the Austroads on-road audit [45] which confirmed that lane departure warning availability was closely related to road class, noting that $98 \%$ of Functional Class 1 roads (major roads) were likely to be supportive of lane keep technology with adequate lane markings, while only $12 \%$ of local minor roads (Class 5) were supportive. Noting that major roads represented $82 \%$ of the network surveyed, the researchers highlighted that primary purpose of the audit was to capture road-readiness on high traffic urban freeways, rural freeways and key rural highways [15].

Similar to road surface quality, the presence of good quality delineation appeared to be more prevalent in major cities. A breakdown of road class by remoteness level showed that of the arterial and sub-arterial roads across remoteness levels, $55 \%$ and $85 \%$ of these 
roads had adequate or low-quality delineation in regional and remote Victoria respectively, while only $36 \%$ of equivalent roads had adequate or low-quality delineation in major cities. None of the sub-arterial roads sampled across the remote regions appeared to have delineation. No literature could be found regarding the relationship between remoteness level and availability of delineated roads in Australasia or globally. While Mackenzie, Dutschke, van den Berg, Kumar and Meuleners [17] assessed the performance of lane support systems on $155 \mathrm{~km}$ of rural highway in Australia, the authors did not acknowledge differences in delineation across road class or remoteness levels, or quantify the availability of delineation.

\subsection{Speed Signage}

Until the development of digital speed maps, improved GNSS-GPS positioning and full cellular coverage across Australia in the future, the functionality of ISA will be highly dependent on the presence and detection of speed signs. Based on the analysis conducted here, speeds signs in remote areas are sporadically placed with large and inconsistent distances between one speed sign and another. Of the 100 locations randomly chosen in remote Victoria, speed signs were present in less than $40 \%$ of the locations, with there being stretches of road greater than $100 \mathrm{~km}$, with no speed sign available. While this is unlikely to be a problem for drivers travelling from one town to another, since speed signs are systematically placed at the entry and exit of most towns, commencing a drive from in-between locations is likely to be highly problematic since vehicles will not be able to detect a speed sign. This, compounded with inconsistent cellular coverage in remote areas [15] presents concerns regarding the availability of ISA supportive infrastructure, a concern echoed by several researchers $[53,54]$.

Conversely, speed sign placement in major cities appeared systematic and thorough. Within major cities, speed signs appeared on all road types at fairly consistent distances from each other, ensuring drivers have to travel no more than two kilometres on average before a speed sign was detected (independent of road class). While this was also the case on regional freeways, drivers had to travel $13.7 \mathrm{~km}$ or $7.4 \mathrm{~km}$ on average before encountering a speed sign on regional and remote highways respectively. Again, these findings are supported by the Austroads on-road audit which found that ISA was unlikely to be a consistently supported technology across Australasian roads [15].

It is noteworthy that drivers in Victoria travelling through unsigned areas are expected to revert to default speed limits imposed by Rule 25 of the Victorian Road safety Road Rules (RSRR (2009), which stipulates a travel speed of $50 \mathrm{~km} / \mathrm{h}$ in built-up areas and $100 \mathrm{~km} / \mathrm{h}$ outside of built-up areas (rural areas). Further, traffic management guides reinforce the notion that no signage is necessary in default speed zones [38]. While the definition of a 'built-up' area is at times not trivial to gauge when driving, it will be more difficult to program into machines and sensors in vehicles which continuously scan the road for detectable speed signs and adapt to the speeds advised on signage. In the absence of such signs, it is likely that either ISA will become ineffective and therefore, ill-trusted or cause confusion for drivers.

\subsection{Overall}

Road quality, delineation presence and speed sign availability across the road classes appeared to decrease with remoteness level. In remote areas, $42 \%$ of roads were low quality (unsealed), $43 \%$ of roads had no delineation with an additional 13.3\% of roads having delineation that only superior lane support systems would likely detect and speed signs were available on only $6 \%$ of sub-arterial, collector and local roads sampled. While this is likely reflective of Victoria's road funding model, where according to the Australian Constitution, roads are the primary responsibility of the state and local governments with support provided federally [55], it is telling of where infrastructure investments should be targeted. 
This methodology also identified that local roads in regional and remote Victoria were consistently lacking the infrastructure measures identified here. These roads are typically funded by councils and given Australia's Councils raise more than $80 \%$ of their own revenue [56], the often reported competing demands on their limited financial resources means that local governments in regional and remote areas are unlikely to be maintained at the standards upheld by roads funded federally or by the state governments. Further to this, of the 537 councils across Australia, close to $55 \%$ are regional, rural or remote councils [57] providing further explanation as to why metropolitan roads are better maintained, but more importantly, justifying the urgent need to review state and federal expenditure if road-readiness for ADAS technologies are to be improved across the nation.

If and when Global Navigation Satellite System (GNSS) systems and high definition maps are available in Australasia, vehicles will be able to estimate their position within a lane and acquire accurate speed sign data almost independently of physical infrastructure [58]. While vehicles which function using these systems are likely to show superior lane keeping performance for example, compared to vision-based systems [58], vision-base lane detection systems are at present fitted to the majority of new vehicles. Having been investigated for over two decades [18], vision-based systems are likely to be relied upon for decades to come and determining the presence and quality of existing physical infrastructure is paramount for ADAS function. Establishing a cost-effective methodology, such as this, which quantifies the availability of such infrastructure, will be critical to targeting efforts where needed so that road-readiness can be improved and the likely benefits of ADAS technologies can be quantified.

\subsection{Study Limitations}

While the method presented here allows for macro-analysis of ADAS-necessary infrastructure availability across road classes, the methodology is not without limitations. First, this methodology is subject to the same limitations which other subjective studies are exposed to. That is, some measures used to quantify road surface quality, delineation quality and speed sign availability are highly dependent on the assessors' judgement. However, the interrater reliability provided assurance that using the protocol, independent reviewers were able to achieve consistent ratings across multiple variables and multiple random locations. Here, Cohen's kappa coefficient was used to estimate interrater reliability and ensured that the possibility of similar agreements occurring between raters were not due to chance.

Limitations were also associated with using aerial imagery alone to quantify road surface quality and delineation quality. While street-view provided high resolution images and therefore, accurate assessments of road surface and delineation quality, street-view was not always available through Nearmap. In such instances, assessments were conducted using only aerial imagery. Poor quality drone images, outdated images, excessive glare, poor weather or roadworks during the time of image capture are likely to have influenced these assessments. Bitumen quality and delineation presence/quality were assessed using aerial imagery alone (in the absence of street-view) for $22 \%$ of sites, potentially preventing an accurate assessment of road and bitumen quality. By conducting a number of assessments of each road class, however, it was expected that errors in judgement due to these reasons were unlikely to have affected the overall findings.

It is important to acknowledge that the purpose of this study was not to determine road readiness for ADAS technologies but rather to develop a methodology that can be used to identify the distribution of roads with adequate ADAS-requiring infrastructure. By applying this methodology to other states and considering more closely the infrastructural needs of ADAS technologies, the road readiness for ADAS technologies can be ascertained and infrastructure investments readily justified. For this reason, characteristics of the infrastructure which may be necessary for quantifying road readiness such as external factors that greatly influence machine detectability of delineation and speed signs (such as road curvature, glare, rain and shadowing, position/angle of signs) were not taken into 
account during the analysis. Instead, roads were assessed objectivity with little regard to how environmental factors would influence, say machine detectability of delineation or speed signs.

\subsection{Future Directions}

This methodology can and should be extended throughout Australasia to identify roads which are not as yet ADAS-ready, and identify where investments into road infrastructure are likely to bring the most gains. While at present infrastructure programs are being fast-tracked to deliver what the federal government considers, 'life-saving' projects using road risk ratings, such as AusRAP, ANRAM and Austroads road stereotype analysis [59], the methodology presented here can also be employed to advise road authorities to invest on roads which are at present, grossly ill-prepared for emerging ADAS technologies. While the science of road surface quality is a complex one [60-66], and should not be undermined, there is no way at present that without using a methodology such as this, that roads can be surveyed without significant financial investment. Further, while Victoria was only used as an example to demonstrate the effectiveness of the methodology, the preparedness of the road network to support AEB, LSS and ISA was not quantified here and likely to be part of a future investigation.

\section{Conclusions}

A robust, cost-effective methodology that uses road class to estimate the availability of infrastructure in preparedness for technologies such as AEB, LSS and ISA is presented here. Using Victoria as an example, the methodology highlighted that roads in major cities and major roads across remoteness levels are high-quality roads that are appropriately delineated and adequately equipped with physical speed signs. Conversely, high-quality roads, delineation and speed signs were severely lacking in regional and remote areas, and particularly on collector and local roads. The findings are consistent with on-road audits and existing CAV trials and reflective of a robust methodology. The results imply that the preparedness of these roads for ADAS technologies should be urgently reviewed and quantified if the benefits of ADAS technologies are to be fully realised.

Author Contributions: Conceptualisation, S.N.; methodology, S.P., J.B.-G., S.N.; data curation, S.P.; investigation, S.P.; formal analysis, S.P., J.B.-G.; resources, J.B.-G., B.F.; writing-original draft preparation, S.P.; writing-review and editing, J.B.-G., S.N., B.F.; supervision, J.B.-G., S.N., B.C., B.F.; project administration, S.P. All authors have read and agreed to the published version of the manuscript.

Funding: This research received no external funding.

Institutional Review Board Statement: Not applicable.

Informed Consent Statement: Not applicable.

Data Availability Statement: Not applicable.

Conflicts of Interest: The authors declare no conflict of interest.

\section{Appendix A. Road Assessment Protocol}

The following protocol was used to rank road surface quality and delineation quality across the road networks in Victoria. Various data were collected and recorded for current and future use.

When answering the following questions, assume that a vehicle fitted with ADAS technologies (AEB and LSS) is travelling in the left-most lane. If the random location is a multilane road, assume the vehicle is travelling straight ahead (i.e., not in a left or right turning lane). Respond assuming that the roadway is reasonably illuminated, and clear/ideal weather conditions are present.

When answering questions about bitumen quality, responses should be provided with respect to a new vehicle travelling on the road at $80 \mathrm{~km} / \mathrm{h}$ being required to apply 
brakes abruptly. Once the responses have been entered for the first direction of travel (D1), enter responses with respect to the opposite direction of travel (D2) by moving virtually to directly across the road. For purposes of simplicity, the variables assessed for only the first direction of travel (D1) are presented here. All responses provided are with respect to a vehicle being driven in Australia (right-hand side drive).

Table A1. The protocol which used for identifing the quality and presence of bitumen and delineation, VIC.

\begin{tabular}{|c|c|}
\hline Variable Name & Description \\
\hline Site_No & Data collection reference/numerical identifier of random location \\
\hline Latitude & Latitude of random location \\
\hline Longitude & Longitude of random location \\
\hline Road_1_Direction & $\begin{array}{c}\text { Position yourself behind/in front of the GPS location marker. Orientate } \\
\text { yourself to the direction of travel. What direction is the traffic } \\
\text { travelling in? } \\
\text { Answer using the Nearmap compass }\end{array}$ \\
\hline Road_Class & $\begin{array}{c}\text { Road class of random location based on the appropriate reference map } \\
\text { and classification used. This will be state-specific. }\end{array}$ \\
\hline
\end{tabular}

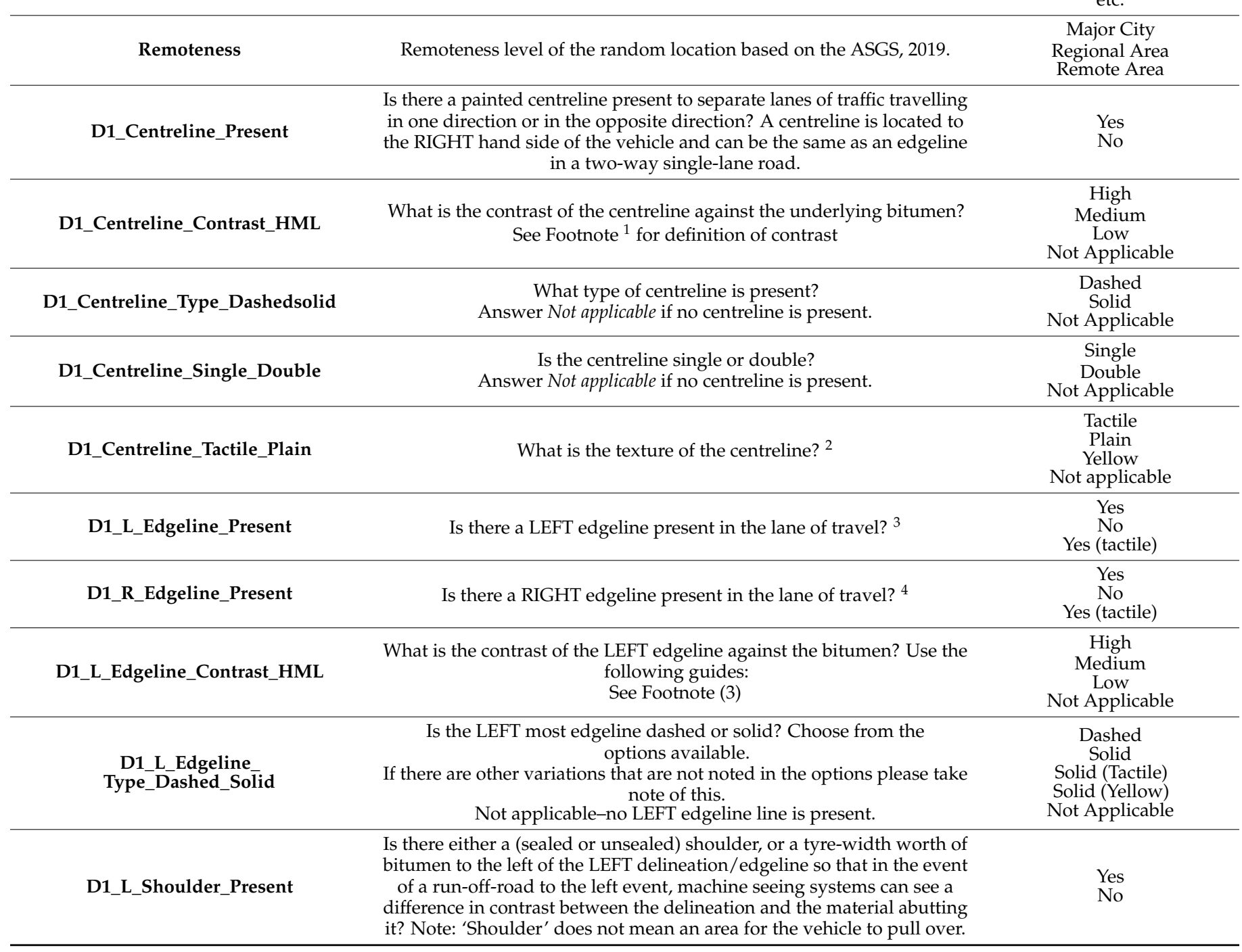


Table A1. Cont.

Variable Name

D1_L_Shoulder_Sealed

D1_L_Shoulder_Substrate

What is the LEFT shoulder composed of? You would choose bitumen if the bitumen extends past the edgeline. Otherwise describe the shoulder composition using the options provided.

Answer Not applicable if no LEFT shoulder is present.

Description
Is the LEFT shoulder sealed or unsealed? ${ }^{5}$
Not applicable-no LEFT shoulder is present.
enter shoulder composed of? You would choose bitumen if
composition using the options provided.
Answer Not applicable if no LEFT shoulder is present.

Is there a properly engineered gutter present? Answer No if culverts or natural drains are present.

This refers to the contrast between the LEFT edgeline and shoulder, OR if there is no LEFT edgeline present, then the contrast between the LEFT gutter and the road. Consider the texture of the material also. If a gutter, shoulder and edgeline are present, answer this with respect to the LEFT edgeline and the abutting material (usually shoulder) ${ }^{6}$

D1_L_Shoulder_
Road_Gutter_Contrast

D1_L_Barrier_Present

Is there a wire rope, concrete barrier or roadside safety barrier present which is likely to prevent a runoff road to the LEFT crash? If there is no such barrier, or if private fencing is present, answer No.

Is there either a (sealed or unsealed) shoulder present, or a tyre-width worth of bitumen to the RIGHT of the RIGHT delineation/edgeline so that in the event of a run-off-road to the right event, machine seeing systems can see a difference in contrast between the delineation and the material abutting it? ${ }^{7}$

D1_R_Shoulder_Present

D1_R_Shoulder_Sealed

Is the RIGHT shoulder sealed or unsealed? ${ }^{8}$

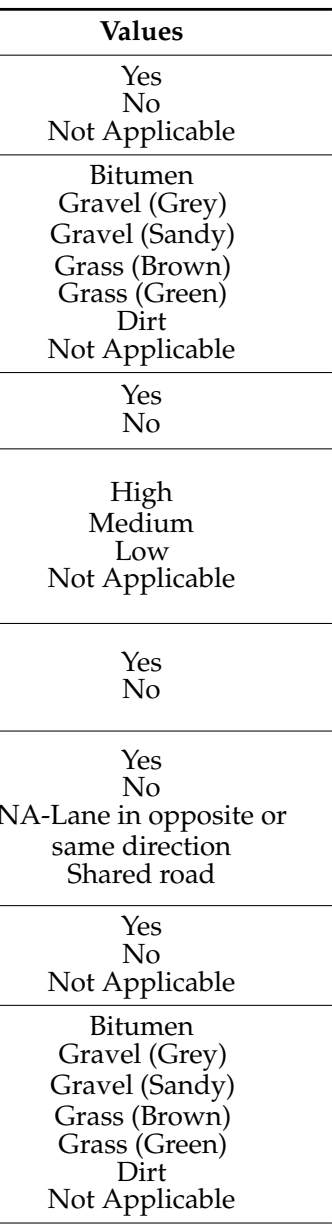

Describe the contrast between the RIGHT edgeline and shoulder, OR if there is no RIGHT edgeline, then the contrast between the gutter and the road. If a gutter, shoulder and edgeline are present, answer this with respect to the RIGHT edgeline and the abutting material (usually shoulder). ${ }^{9}$ Please note that this response should be identical to the D1_L_Edgeline_Contrast_HML if the RIGHT edgeline is equivalent to the centreline.

Description of the right shoulder composition. Choose Bitumen if the bitumen extends past the edgeline. Answer Not applicable if no RIGHT shoulder is present.

$$
\text { Is there a properly engineered gutter present? }
$$

Is there a wire rope, concrete barrier or safety barrier present which is likely to prevent a runoff road to the RIGHT crash? If there is no such barrier, or if private fencing is present, answer No.
High

Medium

Low

Not Applicable
D1_Ex_RIGHT_Gutter

D1_Ex_RIGHT_Barrier

How many lanes of traffic are present for vehicles travelling in the specific direction of travel? Exclude left and right turning lanes and only include the lanes which allow the traffic to travel straight ahead. Answer Shared if the road is unmarked or unseparated and bidirectional traffic are expected to navigate the road.

\section{D1_No_of_Lanes}

\begin{tabular}{cc}
\hline D1_Corregations_Potholes & Are there major defects in the bitumen such as corrugations or potholes. \\
\hline D_1_Sealed_Unsealed? & Is the road sealed or unsealed? Disregard the quality of the seal for \\
this question.
\end{tabular}

${ }^{1}$ High - the contrast of the centreline against the underlying bitumen is extremely high, the centreline appears consistently on the road and is unmissable. Medium - the contrast between the centreline and underlying bitumen is not great, however, the centreline is consistently present and even if faded, likely to be detected by machine seeing systems. Low-there are multiple lines present which can confuse a vehicle (often painted over each other), or the centreline is barely visible or inconsistent in its presence. Not applicable-no centre line is present. ${ }^{2}$ Tactile- the centreline has a intentionally raised texture or has been milled into the bitumen to provide a tactile effect. Plain-the 
pcentreline is a painted white line. Yellow—the centreline is yellow rather than white. Not applicable - no centre line is present. ${ }^{3}$ If there are multiple edgelines present, select the most inner left edgeline (i.e., the one closest to the vehicle as this is what the lane support system will use for lane keeping purposes). Left edgelines can be a centreline of another lane, an edgeline of a bus lane, bike lane, or marked along the gutter. ${ }^{4}$ If there are multiple edgelines present to the right of the vehicle, please select the most inner right edgeline (i.e., the one closest to the vehicle as this is what the lane support system will use for lane keeping purposes). Right edgelines can be a centreline of another lane, an edgeline of a bus lane, bike lane or marked along the gutter. ${ }^{5}$ Sometimes, directly next to the left edgeline is gravel, dirt or other substrate, please note these as being unsealed (answer $\mathrm{No}$ ). If there appears to be at least a tyre-width worth of bitumen to the left of the left edgeline, then this should be considered to be sealed (answer Yes). Moreover, answer Yes if there is another sealed lane to the left, adjoining the lane you are answering questions in reference to. In a multilane road, separated by dashed lines, you would respond Yes if there was an adjoining lane to your left, including bus lane or bike lane. ${ }^{6} \mathbf{H i g h}$ - the gutter and bitumen or left edgeline and shoulder are of drastically contrasting colours (i.e., white against black) or textures. Machine seeing systems should be able to detect a distinct variation between the two surfaces. Medium - there is an adequate amount of contrast/texture and the difference between the gutter and shoulder, or shoulder and left edgeline can be adequately detected based on colour and texture alone. Low-the gutter and the bitumen or shoulder and left edgeline are almost identical colours and textures. If the shoulder falls away from the bitumen, or rises above the level of the bitumen and left edgeline marking, select Low as machine seeing systems require the delineation (and the gutter or shoulder if relying on these) to be in level with the travel lane. Not applicable-no shoulder or gutter is present next to the left delineation.

${ }^{7}$ Note that in this instance, the term 'Shoulder' does not mean an area for the vehicle to pull over. If the right edgeline is equivalent to a centreline, then answer Not applicable - Lane in opposite or same direction. Choose Shared road if the road is not separated by dashed or solid delineation i.e., a completely unmarked road. ${ }^{8}$ Sometimes, directly next to the right edgeline is gravel, dirt or other substrate, please note these as being unsealed (answer No). If there appears to be at least a tyre-width worth of bitumen to the right of the right edgeline, then this should be considered to be sealed (answer Yes). Answer Yes if there is another sealed lane to the right, adjoining the lane you are answering questions in reference to. In a multilane road, separated by dashed lines, you would respond Yes if there was an adjoining lane to the right. Answer Not applicable if no right shoulder is present. ${ }^{9} \mathrm{High}-$ the gutter and bitumen or right edgeline and shoulder are of drastically contrasting colours (i.e., white against black) and textures. Machine seeing systems should be able to detect a distinct variation between the two surfaces. Medium — there is an adequate amount of contrast/texture and the difference between the gutter and shoulder, or shoulder and right edgeline can be adequately detected based on colour and texture alone. Low—the gutter and the bitumen or shoulder and right edgeline are almost identical colours or textures. If the shoulder falls away from the bitumen, or rises above the level of the bitumen and right edgeline marking, select Low as machine seeing systems require the delineation (and the gutter or shoulder if relying on these) to be in level with the lane of travel. Not applicable-no shoulder or gutter is present next to the right delineation. ${ }^{10}$ High - the bitumen is intact with no defects visible. The surface looks relatively well maintained and there appears to be good traction between tyres of a new vehicle and the road. In the event of unanticipated sudden breaking, the vehicle is unlikely to skid due to poor surface quality. Medium—bitumen is intact however, there may be alligator or/and longitudinal cracks and general defects along the length of the road. These include longitudinal depressions in wheel path, transverse undulations of pavement surface and visible friction variations. It is more than likely that in the event of sudden breaking, the vehicle will be able to stop without skidding. Low-poor quality roads where the bitumen is not consistently intact, there are pavement drop-offs, potholes or the road is only partially sealed or unsealed. It is more than likely that in the event of sudden breaking, the vehicle will not be able to stop without skidding.

\section{References}

1. Treat, J.R.; Tumbas, N.; McDonald, S.; Shinar, D.; Hume, R.D.; Mayer, R.; Stansifer, R.; Castellan, N. Tri-Level Study of the Causes of Traffic Accidents: Final Report. Executive summary; Institute for Research in Public Safety, Indiana University: Bloomington, IN, USA, 1979; p. 82.

2. Dingus, T.A.; Guo, F.; Lee, S.; Antin, J.F.; Perez, M.; Buchanan-King, M.; Hankey, J. Driver crash risk factors and prevalence evaluation using naturalistic driving data. Proc. Natl. Acad. Sci. USA 2016, 113, 2636-2641. [CrossRef]

3. Otte, D.; Pund, B.; Jänsch, M. A new approach of accident causation analysis by seven steps ACASS. In Proceedings of the 21st International Technical Conference on the Enhanced Safety of Vehicles (ESV), Stuttgart, Germany, 15-18 June 2009.

4. NHTSA. National Motor Vehicle Crash Causation Survey: Report to Congress; U.S. Department ofTransportation, National Highway Traffic Safety Administration: Springfield, VA, USA, 2008; p. 059.

5. Lijarcio, I.; Useche, S.A.; Llamazares, J.; Montoro, L. Availability, demand, perceived constraints and disuse of ADAS technologies in Spain: Findings from a national study. IEEE Access 2019, 7, 129862-129873. [CrossRef]

6. Lubbe, N.; Jeppsson, H.; Ranjbar, A.; Fredriksson, J.; Bärgman, J.; Östling, M. Predicted road traffic fatalities in Germany: The potential and limitations of vehicle safety technologies from passive safety to highly automated driving. In Proceedings of the IRCOBI Conference, Athena, Greece, 12-14 September 2018.

7. Milakis, D.; Van Arem, B.; Van Wee, B. Policy and society related implications of automated driving: A review of literature and directions for future research. J. Intell. Transp. Syst. 2017, 21, 324-348. [CrossRef] 
8. Hynd, D.; McCarthy, M.; Carroll, J.; Seidl, M.; Edwards, M.; Visvikis, C.; Tress, M.; Reed, N.; Stevens, A. Benefit and Feasibility of a Range of New Technologies and Unregulated Measures in the Fields of Vehicle Occupant Safety and Protection of Vulnerable Road Users; 9279446622; Report to the European Commission; Luxembourg Publications Office: Luxembourg, 2015.

9. Winkle, T. Development and approval of automated vehicles: Considerations of technical, legal, and economic risks. In Autonomous Driving; Springer: Berlin, Germany, 2016; pp. 589-618.

10. Rolison, J.J.; Regev, S.; Moutari, S.; Feeney, A. What are the factors that contribute to road accidents? An assessment of law enforcement views, ordinary drivers' opinions, and road accident records. Accid. Anal. Prev. 2018, 115, 11-24. [CrossRef] [PubMed]

11. ANCAP. Availability of Autonomous Emergency Braking (AEB) in Australia; ANCAP: Manuka, ACT, Australia, 2020.

12. 76 Robson, R. Availability of Safety Features as Standard Inclusions; Australasian New Car Assessment Program (ANCAP): Manuka, ACT, Australia, 2020; p. 2.

13. Peiris, S.; Berecki-Gisolf, J.; Chen, B.; Fildes, B. Road Trauma in Regional and Remote Australia and New Zealand in Preparedness for ADAS Technologies and Autonomous Vehicles. Sustainability 2020, 12, 4347. [CrossRef]

14. Evans, J. Intelligent speed assistance: Everything you need to know. AutoCar 2019. Available online: https://www.autocar.co.uk/ car-news / features/intelligent-speed-assistance-everything-you-need-know (accessed on 27 May 2021).

15. Germanchev, A.; Eastwood, B.; Hore-Lacy, W. Infrastructure Changes to Support Automated Vehicles on Rural and Metropolitan Highways and Freeways Road Audit (Module 2); Austroads Ltd.: Sydney, NSW, Australia, October 2019; p. 90.

16. Goodin, C.; Carruth, D.; Doude, M.; Hudson, C. Predicting the Influence of Rain on LIDAR in ADAS. Electronics 2019, 8, 89. [CrossRef]

17. Mackenzie, J.; Dutschke, J.; van den Berg, A.; Kumar, M.; Meuleners, L. Automated Vehicles and the Readiness of Western Australian Roads; Curtin University: Bentley, WA, Australia, 2018.

18. Narote, S.P.; Bhujbal, P.N.; Narote, A.S.; Dhane, D.M. A review of recent advances in lane detection and departure warning system. Pattern Recognit. 2018, 73, 216-234. [CrossRef]

19. Pape, D.; Habtemichael, F. Infrastructure Initiatives to Apply Connected-and Automated-Vehicle Technology to Roadway Departures; U.S Department of Transportation, Federal Highway Administration: McLean, VA, USA, 2018; pp. 1-108.

20. Raj, A.C.; Sozio, A.; Velden, M.V.d. Guidance and Readability Criteria for Traffic Sign Recognition Systems Reading Electronic Signs; Austroads: Sydney, NSW, Australia, 2020; p. 57.

21. National Transport Commission. Guidelines for Trials of Automated Vehicles in Australia; National Transport Commission: Melbourne, VIC, Australia, 2020; p. 24.

22. Austroads. Trials. Available online: https://austroads.com.au/drivers-and-vehicles/future-vehicles-and-technology/trials (accessed on 25 March 2021).

23. Gunner, M. New Era of Modern Travel to Hit Waterfront. Available online: https://newsroom.nt.gov.au/mediaRelease/22676 (accessed on 23 March 2021).

24. Moore, T. Ipswich Set to Trial Electric Driverless Shuttle Buses. Available online: https://www.smh.com.au/business/smallbusiness / ipswich-set-to-trial-electric-driverless-shuttle-buses-20180221-p4z145.html (accessed on 23 March 2021).

25. Transport for NSW Regional Automated Vehicle Trials. Available online: https://www.transport.nsw.gov.au/projects / programs / smart-innovation-centre/regional-automated-vehicle-trials\#Armidale (accessed on 23 March 2021).

26. RAC Experience the Future Today. Available online: https://rac.com.au/about-rac/advocating-change/initiatives/automatedvehicle-program/intellibus (accessed on 23 March 2021).

27. Department for Infrastructure and Transport Driverless Vehicles (Details of Approved Trials). Available online: https://www.dpti. sa.gov.au/driverlessvehicles (accessed on 25 March 2021).

28. Queensland Government Ipswich Connected Vehicle Pilot. Available online: https://www.qld.gov.au/transport/projects/cavi/ ipswich-connected-vehicle-pilot (accessed on 23 March 2021).

29. VicRoads. VicRoads-ITS Grants Program: Connected and Automated Vehicle Technology. Available online: https://www. vicroads.vic.gov.au/safety-and-road-rules/vehicle-safety/automated-and-connected-vehicles (accessed on 23 March 2021).

30. Somers, A. Infrastructure Changes to Support Automated Vehicles on Rural and Metropolitan Highways and Freeways: Audit Specification (Module 1); AP-T347-19; Austroads: Sydney, NSW, Australia, October 2019; pp. 1-44.

31. Transurban. NSW Partially Automated Vehicle Trials. Stage one-Sydney Orbital Network; Transurban: Sydney, NSW, Australia, 2018; p. 26.

32. Transurban. Victorian Connected and Automated Vehicle Trials Program: Phase One; Transurban: Melbourne, VIC, Australia, 2018; p. 26.

33. Victorian Government First Automated Vehicle To Hit The Road. Available online: https://www.premier.vic.gov.au/firstautomated-vehicle-hit-road (accessed on 25 March 2021).

34. SAE. European Union Approves Mandatory Vehicle Speed-Limiting Feature. Available online: https://www.sae.org/news/2019 /04/eu-to-mandate-intelligent-speed-assistance (accessed on 23 March 2021).

35. Australian Bureau of Statistics. Australian Statistical Geography Standard (ASGS): Volume 5-Remoteness Structure. 2019. Available online: https:/ / www.abs.gov.au/ausstats/abs@.nsf/mf/1270.0.55.005 (accessed on 13 September 2019).

36. Data.vic.gov.au Digital Road Network Map_Vicmap Transport (“TR_Road”). Available online: http://services.land.vic.gov.au/ catalogue/metadata?anzlicId=ANZVI0803002595\&publicId=guest\&extractionProviderId=1\#tab1 (accessed on 9 January 2021).

37. TNO. Human Factors Guidelines Report 2: Driver Support Systems Overview; TNO: Helmond, The Netherlands, February $2021 ;$ p. 62. 
38. VicRoads. Traffic Engineering Manual: Volume 3-Additional Network Standards \& Guidelines. In ANSEG Part 2.11: Speed Zoning Guidelines, 1st ed.; VicRoads: Victoria, Australia, 2017; pp. 1-98.

39. EuroNCAP. EuroNCAP Test Protocol-Lane Support Systems; Version 3.0.2; European New Car Assessment Programme (EuroNCAP): Leuven, Belgium, 2019; pp. 1-25.

40. ANCAP. Today's Tech-Lane Support Systems: Let's Re-Write the Ending; Australasian New Car Assessment Program (ANCAP): Manuka, ACT, Australia, 2021; p. 2.

41. DataVIC. Speed Sign Data; VicRoads (Department of Transporation): Victoria, Australia, 2021.

42. McHugh, M.L. Interrater reliability: The kappa statistic. Biochem. Med. 2012, 22, 276-282. [CrossRef]

43. Austroads. Future Vehicles 2030; Austroads Project No. FCA6239; Austroads: Sydney, NSW, Australia, June 2020; pp. 1-105.

44. Austroads. Infrastructure Changes to Support Automated Vehicles on Rural and Metropolitan Highways and FreewaysAudit Specification (Module 1); Austroads: Sydney, NSW, Australia, October 2019; p. 39.

45. Somers, A. Infrastructure Changes to Support Automated Vehicles on Rural and Metropolitan Highways and Freeways: Project Findings and Recommendations (Module 5); 1925854434; Austroads Ltd.: Sydney, NSW, Australia, 8 October 2019; p. 67.

46. McLean, J.; Foley, G. Road Surface Characteristics and Condition Effects of Road Users; ARRB Transport Research Ltd.: Vermont South, VIC, Australia, 1998; p. 55.

47. Verity, S.; Mellor, A. National State of the Assets 2018; Australian Local Government Association: Deakin, ACT, Australia, 2018; p. 101.

48. BITRE. Australian Infrastructure Statistics Yearbook 2020; Bureau of Infrastructure and Transport Research Economics: Canberra, Australia, 2020; p. 328.

49. IPWEA. The International Infrastructure Management Manual, 5th ed.; Institute of Public Works Engineering Australasia: Wellington, New Zealand, 2015; p. 88.

50. VicRoads. Guide to Surface Inspection Rating: For Pavements Surfaced with Sprayed Seals and Asphalt; VicRoads: Victoria, Australia, September 2009; p. 62.

51. FHWA. Manual on Uniform Traffic Control Devices 2009; US DOT Federal Highway Administration: Washington, DC, USA, 2009; p. 864.

52. Ratingen, M.V. Lane Keep Assist Technology. Personal Communication; European New Car Assessment Programme (EuroNCAP): Leuven, Belgium, 26 March 2021.

53. Herranz-Perdiguero, C.; López-Sastre, R.J. ISA2: Intelligent Speed Adaptation from Appearance. arXiv 2018, arXiv:1810.05016.

54. Unger, T.; Schubert, A. Qualitative Assessment of Intelligent Speed Adaptation (ISA) Systems; Verkehrsunfallforschung an der TU Dresden GmbH_VUFO: Dresden, Germany, 22 October 2018; p. 47.

55. Buchanan, K. National Funding of Road Infrastructure: Australia; Library of Congress, Law Library of Congress: Washington, DC, USA, 2014.

56. Australian Local Government Association. Roads Funding. Available online: https://alga.asn.au/policy-centre/roads-andinfrastructure/roads-funding/ (accessed on 21 April 2021).

57. Australian Local Government Association. Local Government Key Facts and Figures. Available online: https://alga.asn.au/ facts-and-figures/ (accessed on 30 April 2021).

58. Tominaga, K.; Takeuchi, Y.; Tomoki, U.; Kameoka, S.; Kitano, H.; Quirynen, R.; Berntorp, K.; Cairano, S. GNSS Based Lane Keeping Assist System via Model Predictive Control; 0148-7191; SAE Technical Paper; SAE: Warrendale, PA, USA, 2019.

59. ARRB. Proactive Mass-Action Road Safety Treatments across Regional Australia. Available online: https://www.arrb.com. $\mathrm{au} /$ news / proactive-mass-action-road-safety-treatments-across-regional-australia?utm_campaign=iNTRO\&utm_medium= email\&_hsmi=101526207\&_hsenc=p2ANqtz--ZZI8mqjWrCBXItOnyytUwP3B0vkqq2yQ4VvZhbm8rYueA_16TyeIsbnfLI_N1BJ4 pwkN1F5vbYb159vog37kE8XhOkTduJkBi_DfATJD1h5RK8vw\&utm_content=101526207\&utm_source=hs_email (accessed on 16 December 2020).

60. Potter, D. Measurement of Road Roughness in Australia. In Transportation Research Record; National Academies of Sciences, Engineering, and Medicine: Washington, DC, USA, 1978.

61. VicRoads. Technical Note 50: GeoPave. In Ride Quality of Pavements; VicRoads: Victoria, Australia, $2002 ;$ p. 2.

62. McLean, J.; Ramsay, E. Interpretations of Road Profile Roughness Data: Review and Research Needs; ARRB Transport Research Ltd.: Vermont South, VIC, Australia, 1996.

63. Carteret, R.D.; Comport, L.; Metcalf, J.; Rebbechi, J. Guide to Pavement Technology Part 8: Pavement C; Austroads Ltd.: Sydney, NSW, Australia, 2009.

64. Jameson, G. Guide to Pavement Technology Part 5: Pavement Evaluation and Treatment Design; Austroads Ltd.: Sydney, NSW, Australia, 2011.

65. Vorobieff, G. Guide to Pavement Technology Part 8: Pavement Construction; Austroads Ltd.: Sydney, NSW, Australia, 2009 ; p. 243.

66. Choummanivong, L.; Martin, T. Long-Term Pavement Performance Study: Final Report; Austroads Ltd.: Sydney, NSW, Australia, January 2019; p. 82. 